\title{
Évolution démographique du Saguenay-Lac-Saint-Jean : tendances récentes, situation actuelle et perspectives d'avenir
}

\author{
Marc Tremblay et Hélène Vézina ${ }^{1}$ \\ Université du Québec à Chicoutimi
}

\section{Introduction}

En 2003, la population de la région du SaguenayLac-St-Jean (SLSJ) s'élevait à 278519 individus ${ }^{2}$. Après avoir atteint un sommet de plus de 292000 personnes vers la fin des années 1980, cette population décroît à un rythme qui s'est accentué au cours des dernières années. Quels sont les principaux facteurs qui expliquent cette diminution de la taille de la population? S'agit-il de facteurs qui sont spécifiques au SLSJ ou à l'ensemble des populations des "régions », ou alors caractérisent-ils le Québec, voire le monde industrialisé dans son entier? Quelles sont les conséquences démographiques de cette décroissance à court et à moyen terme ? Ces effets sont-ils irréversibles ou est-il encore possible de contrer au moins certains d'entre eux ? Le cas échéant, comment faut-il intervenir ? Est-il souhaitable de le faire? Dans cet article, deux démographes du Groupe de recherche interdisciplinaire en démographie et épidémiologie génétique de l'Université du Québec à Chicoutimi se proposent de fournir au lecteur non pas des réponses définitives à ces questions, mais des données et des considérations qui lui permettront d'alimenter ses réflexions sur un sujet qui concerne l'ensemble des citoyens de la région.

\section{Bref historique du peuplement : des milliers d'immigrants et une natalité vigoureuse}

C'est autour de 1835 que la région du SLSJ a été ouverte au peuplement d'origine européenne et, au recensement de 1852, on y dénombrait déjà 5364 habitants ${ }^{3}$. Contrairement à certaines croyances, ce ne sont donc pas une poignée d'immigrants qui sont à l'origine du peuplement initial de la région, mais plusieurs milliers. En effet, entre 1838 et 1911, environ 30000 immigrants sont venus s'établir au SLSJ. Jusqu'en 1870 , près de $80 \%$ de ces pionniers étaient originaires de la région de Charlevoix mais, par la suite, cette proportion baisse à moins de $50 \%$ alors que l'origine des immigrants se diversifie. On sait cependant que les immigrants de Charlevoix ont été plus nombreux à s'installer définitivement dans la région et ont réussi à y implanter un plus grand nombre de leurs descendants, ce qui a eu pour effet d'accentuer leur part dans la formation de la population saguenayenne, notamment sur le plan génétique ${ }^{4}$.

Malgré un fort contingent d'immigrants, les mouvements de population à l'échelle régionale comportent, dès 1880 , plus de sorties que d'entrées et se soldent par un bilan migratoire le plus souvent négatif. C'est donc essentiellement sur une natalité vigoureuse qu'a reposé la croissance de la population. Cette croissance est d'ailleurs plutôt impressionnante puisque, entre 1861 et 1961, la taille de la population est multipliée par 25 pour passer de 10478 à 262426 . À titre comparatif, la population du Québec, pendant la même période, a seulement quintuplé. La fécondité demeure élevée jusqu'au début des années 1960, alors qu'elle est encore à plus de 4 enfants par femme en moyenne. Le déclin, amorcé un peu plus tardivement qu'ailleurs au Québec, s'effectue de façon très rapide et, en 1986, la fécondité au SLSJ (1,5 enfant) est à peine plus élevée que la moyenne québécoise qui est passée à 1,4 enfant par femme. La population continue cependant à croître, bien que moins rapidement, jusqu'au milieu des années 1980. 


\section{Évolution récente de la situation \\ démographique : décroissance et perte de poids}

La décroissance de la population du SLSJ a commencé au début des années 1990 à un rythme d'abord très lent, soit $-0,1 \%$ par année de 1991 à 1996, puis un peu plus rapide, soit $-0,6 \%$ annuellement entre 1996 et 2003 (figure 1). Au $1^{\mathrm{er}}$ juillet 2003, la population du SLSJ comportait 278519 individus répartis à peu près également entre les deux sexes. Si l'on regarde la pyramide des âges de la population (figure
2), on constate cependant que la distribution selon le sexe varie selon les groupes d'âge. Comme dans la plupart des populations contemporaines, le nombre d'individus de sexe masculin est plus élevé dans les groupes d'âge les plus jeunes. Ceci s'explique par le fait qu'il naît en moyenne 105 garçons pour 100 filles et que les garçons conservent l'avantage numérique qui leur est ainsi conféré jusqu'à la mi-quarantaine environ. Par la suite, la situation s'inverse progressivement à cause d'une surmortalité masculine et d'une espérance de vie plus élevée pour les femmes. Ainsi, chez les 85 ans et plus, on ne retrouve plus que 43 hommes pour 100 femmes.

Figure 1 - Population du Saguenay-Lac-Saint-Jean, 1986-2026 (projections après 2001)

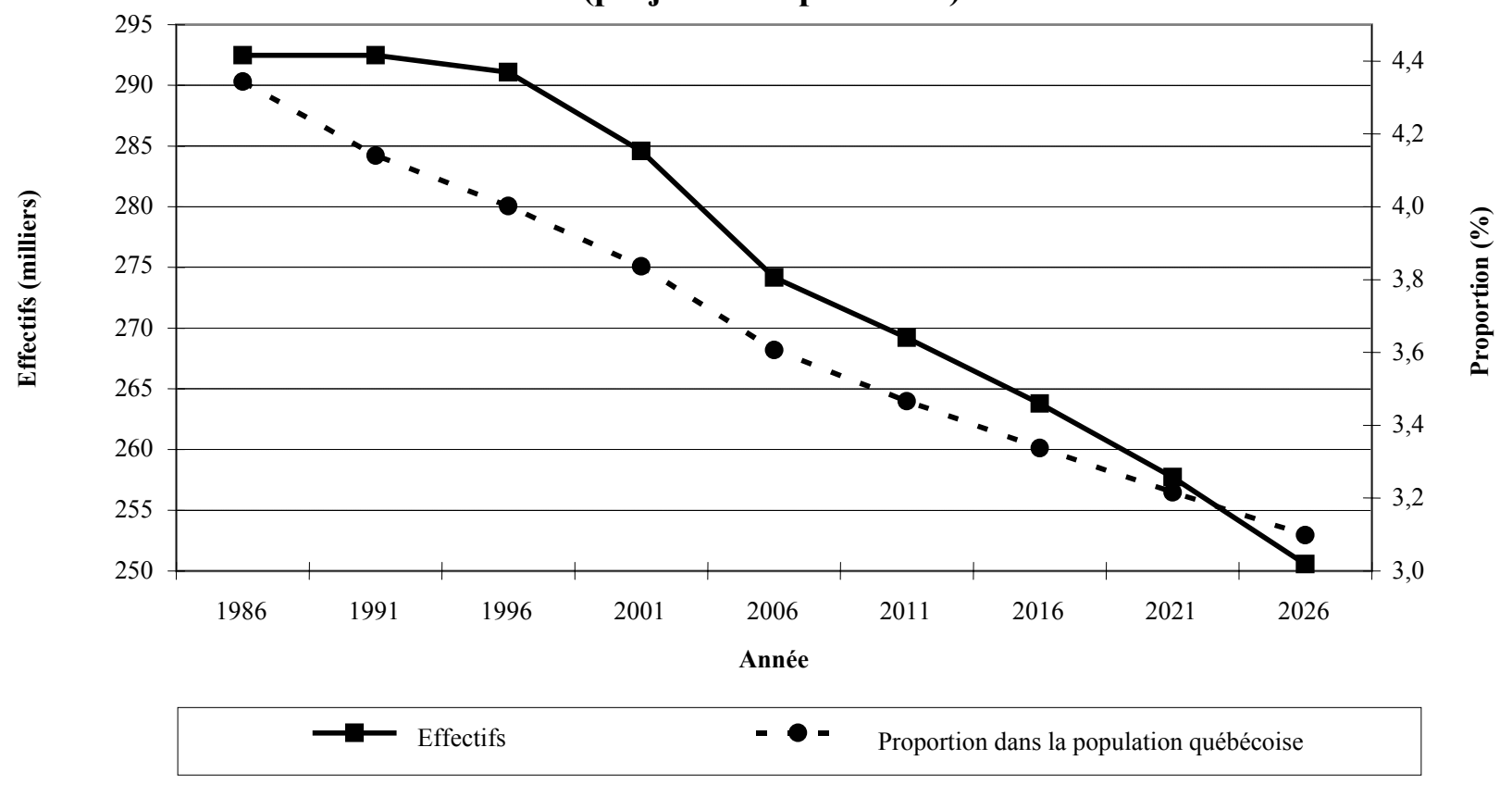

La pyramide de la figure 2 nous montre aussi qu'il devient de plus en plus difficile de comprendre d'où ce type de représentation graphique tire son nom. La forme pyramidale subsiste pour la population âgée de plus de 40 ans, mais on doit reconnaître que cette pyramide repose sur un socle de forme plutôt irrégulière. Les groupes d'âge les plus nombreux sont ceux dont la période de naissance se situe entre 1948 et 1963 (les baby-boomers). Pour les groupes âgés de moins de 25 ans, soit les individus nés depuis 1978, on peut presque parler de pyramide inversée, avec des effectifs de moins en moins nombreux chez les plus jeunes. Ceci s'observe, quoiqu'à des degrés divers, dans tous les pays européens et nord-américains qui ont vécu le baby-boom de l'après-guerre. Enfin, le creux observé chez les individus nés entre 1963 et 1978, et donc âgés de 25 à 39 ans en 2003, s'explique à la fois par la baisse importante de fécondité observée pendant cette période et par l'émigration de plusieurs individus de ce groupe. Mis à part ce creux, la structure par âge de la population saguenayenne est semblable à celle de la population québécoise. 


\section{Figure 2 - Pyramide des âges de la population du Saguenay-Lac-Saint-Jean,}

$1^{\text {er }}$ juillet 2003

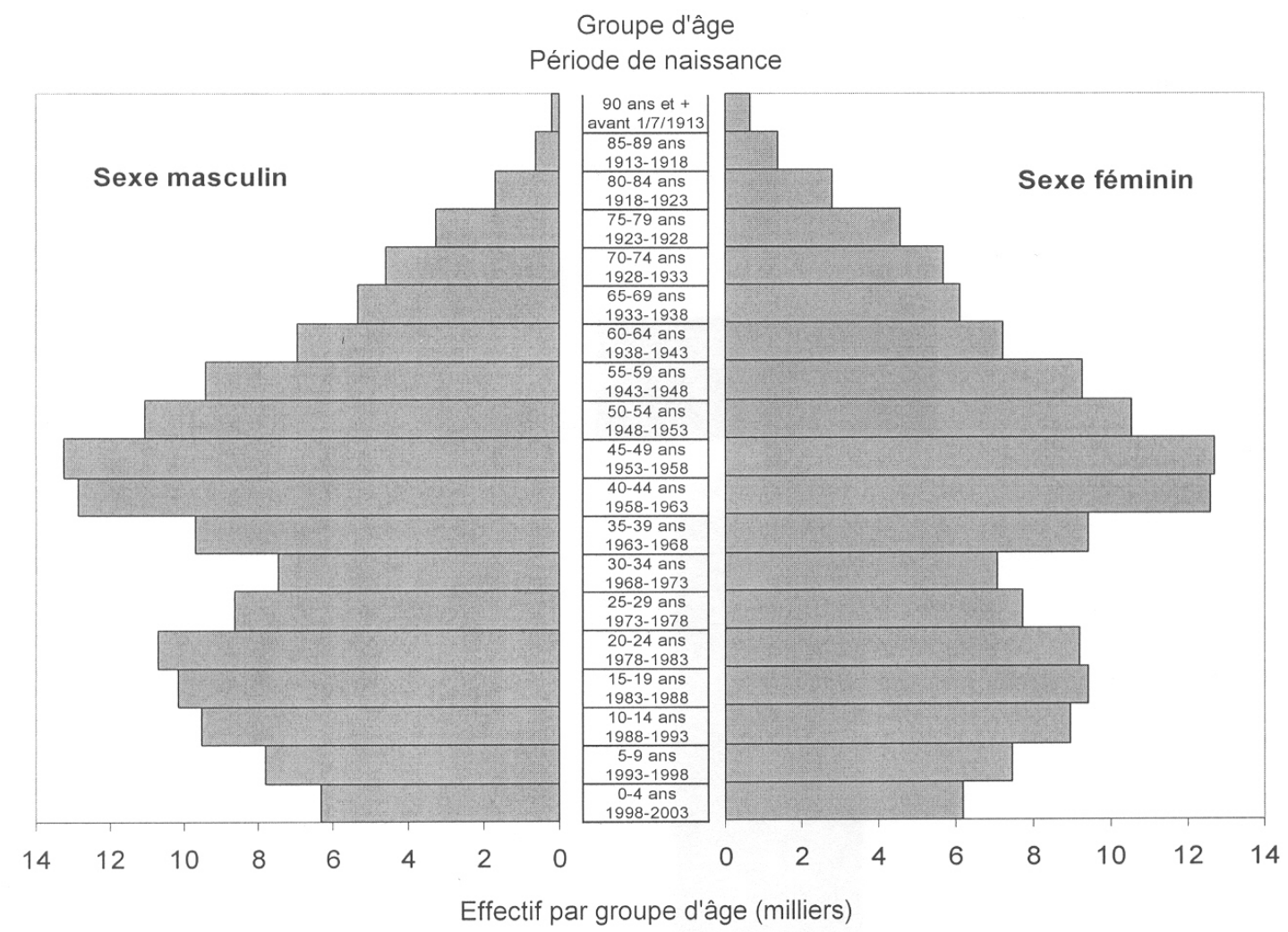

À l'échelle provinciale, une des conséquences immédiates de la baisse de population au SLSJ est la diminution du poids démographique de la région dans l'ensemble du Québec qui est passé de 4,3 \% en 1986 à $3,8 \%$ en 2003 (figure 1). En effet, l'évolution démographique récente a été extrêmement variable d'une région à l'autre, comme en témoignent les données présentées à la figure 3 qui montre les 17 régions administratives classées selon l'importance de leur croissance relative de 1991 à 2003. On y constate que la croissance s'effectue selon une concentration géographique marquée. Les régions de l'ouest de la province, et plus particulièment celles situées au nord de l'île de Montréal, connaissent les plus fortes croissances. La région des Laurentides se démarque nettement de toutes les autres avec une augmentation de $25 \%$ de sa population pour la période considérée. Les régions du centre du Québec connaissent aussi une croissance positive mais plus modérée (entre $3 \%$ et $8 \%$ ). C'est aussi dans cette catégorie que se situent l'île de Montréal et le Nord du Québec. Enfin, les ré- gions du nord et de l'est de la province vivent dans un contexte de croissance négative qui s'amplifie avec l'éloignement des grands centres, alors qu'on observe une diminution des populations de ces régions allant de $2 \%$ à $10 \%$ au cours des douze dernières années.

\section{L'accroissement naturel : de moins en moins de naissances, de plus en plus de décès}

La chute de la fécondité amorcée dans les années 1960 a eu comme conséquence de faire baisser rapidement le nombre de naissances dans la région. À la fin des années 1980, il y avait déjà deux fois moins de naissances qu'à la fin des années 1950, malgré la plus grande taille de la population. Cette baisse des naissances, conjuguée à la hausse du nombre de décès, explique la forte diminution de l'accroissement naturel (différence entre le nombre annuel de naissances et le nombre annuel de décès) au cours des 20 dernières années (figure 4). 


\section{Figure 3 - Croissance relative (\%) des populations régionales du Québec, 1991-2003}

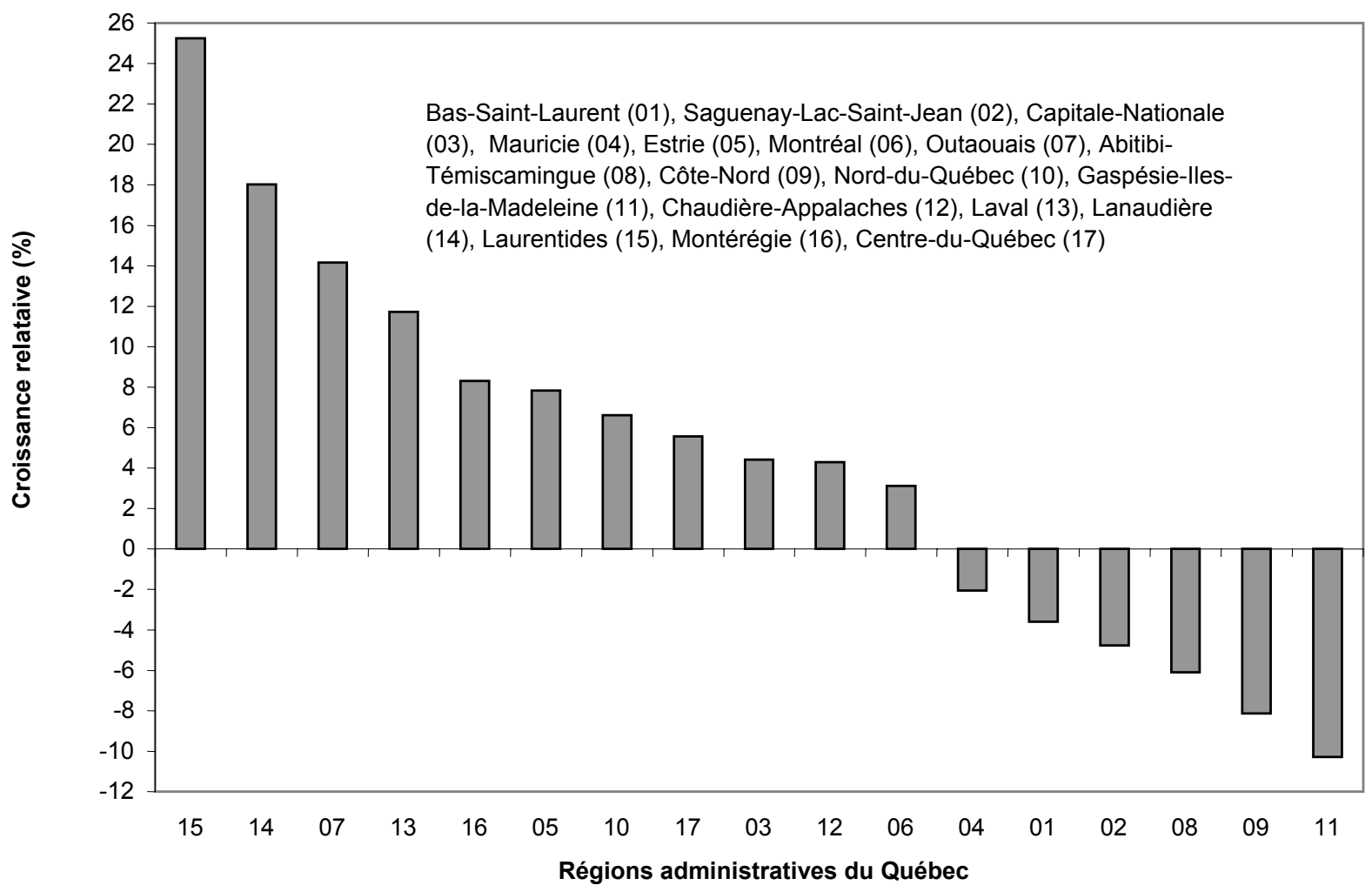

La mesure la plus fréquemment utilisée pour illustrer la fécondité d'une population est l'indice synthétique de fécondité (exprimé en termes de nombre moyen d'enfants par femme), qui résume les conditions de fécondité observées dans une population durant une année donnée. Cet indice est présentement d'environ 1,5 enfant par femme dans la population du SLSJ. Après avoir frôlé la valeur de 1,4 enfant par femme en 1988, l'indice a remonté quelque peu au début des années 1990 (jusqu'à 1,7), mais depuis 1999, il fluctue entre 1, 4 et 1,5. Ces valeurs ne sont pas suffisamment élevées pour assurer le remplacement des générations puisqu'il faut, pour ce faire, un minimum de 2,1 enfants par femme.

Malgré les faibles valeurs de l'indice synthétique de fécondité, la population a tout de même maintenu un accroissement naturel positif jusqu'à maintenant. En effet, il y encore un peu plus de naissances que de décès à chaque année dans la population. Ceci s'explique par la structure par âge de la population qui fait en sorte que les générations en âge d'avoir des enfants sont encore assez nombreuses pour compenser leur faible fécondité. Cependant, la population vieillit, et puisque les risques de décès augmentent avec l'âge, le nombre de décès est présentement en hausse. Cette hausse des décès est inéluctable, malgré l'augmentation de l'espérance de vie à la naissance (nombre moyen d'années à vivre, selon les conditions de mortalité observées dans la population). L'espérance de vie a en effet augmenté d'à peu près trois ans dans la région depuis 1986 (de 71 à 74 ans chez les hommes et de 78 à 81 ans chez les femmes), mais ici encore, les effets de structure par âge se font sentir : le nombre de personnes atteignant les groupes d'âge avancés (là où les risques de décès sont les plus élevés) étant de plus en plus nombreux, cela ne peut que faire augmenter le nombre de décès à chaque année. 
Figure 4 - Naissances, décès et accroissement naturel dans la population du Saguenay-Lac-Saint-Jean, 1986-2025 (projections après 2003)

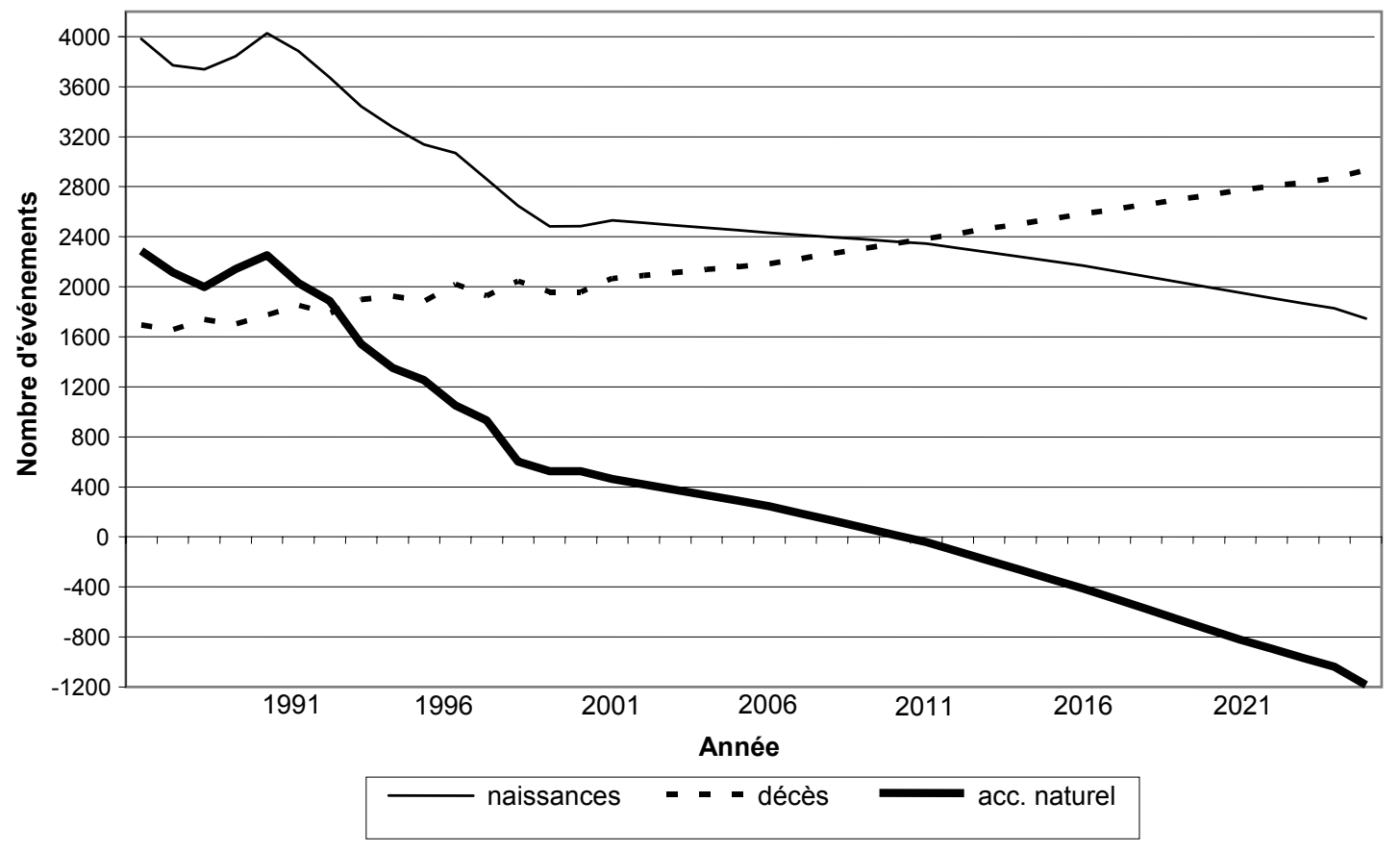

Durant l'année 2003, environ 2400 naissances ont été enregistrées dans la région du SLSJ, pour un taux de natalité (rapport du nombre de naissances à la population moyenne) de 8,7 pour 1000 . Ceci représente une baisse de $40 \%$ par rapport à l'année 1990 (4000 naissances et taux de 13,7 pour 1000$)$. Le taux de natalité régional se situe un peu au-dessous de la moyenne provinciale (9,8 pour 1000 en 2003). Durant la même période, le nombre de décès passait de 1800 à environ 2100 (nombre estimé), correspondant à des taux de mortalité de 6,1 pour 1000 (1990) et 7,6 pour 1000 (2003). Depuis quelques années, les valeurs du taux de mortalité saguenayen ont rattrapé et même dépassé légèrement celles de l'ensemble de la population québécoise (7,4 pour 1000 en 2003).

En somme, l'accroissement naturel qui, pendant des décennies, a assuré la croissance de la population saguenayenne se dirige lentement mais sûrement vers des valeurs négatives. En 2003, il n'était plus que de 300 .

\section{Les migrations : facteur de décroissance régionale}

Rares sont les populations qui ne sont pas affectées d'une manière ou d'une autre par les mouvements mi- gratoires. En fait, les migrations constituent souvent une composante déterminante du renouvellement des populations. Selon la direction et l'importance que prennent ces mouvements migratoires, on peut classer les populations selon deux grandes catégories : celles qui profitent de ces mouvements (les populations " gagnantes »), en ce sens que les migrations contribuent à leur croissance, au détriment des autres populations (les populations "perdantes») qui voient partir un plus grand nombre d'individus qu'ils en accueillent. La population actuelle de la région du SLSJ appartient à cette seconde catégorie.

Évidemment, cette classification plutôt grossière appelle quelques nuances. En effet, les mouvements migratoires ne vont pas nécessairement toujours dans le même sens, et une même population peut subir des pertes durant une certaine période et profiter de gains substantiels par la suite. Dans le cas du SLSJ, les périodes les plus «fastes » sur le plan migratoire ont été, clairement, celles du début de la colonisation avec l'arrivée des pionniers en provenance de Charlevoix. Dès la fin du $19^{\mathrm{e}}$ siècle, cependant, la région avait déjà commencé à fournir des émigrants aux centres urbains de Québec et Montréal, ainsi qu'aux ÉtatsUnis ${ }^{5}$. À part quelques épisodes éphémères liés à l'implantation des grandes industries (Price, Alcan), la 
plus grande partie du $20^{\mathrm{e}}$ siècle a été caractérisée par des pertes migratoires pour la région. La population a cependant continué à croître grâce, nous l'avons vu, à son accroissement naturel élevé. Cette situation devait toutefois changer rapidement durant le dernier quart du siècle.

La chute brutale de la fécondité a fait en sorte que l'importance relative de la migration a pris de l'ampleur depuis une trentaine d'années. Les pertes migratoires, autrefois nettement compensées par l'accroissement naturel, sont devenues le principal moteur de la décroissance démographique dans la région. Ces pertes résultent principalement des échanges migratoires avec les autres régions de la province (le solde migratoire interrégional). Les deux autres types de mouvements migratoires, soit les migrations interprovinciales (entrants et sortants interprovinciaux) et internationales (immigrants et émigrants internationaux), sont d'une ampleur beaucoup plus faible et n'ont que peu d'impact sur le renouvellement de la population régionale (figure 5). Ainsi, depuis 1986-
1987, le solde migratoire interrégional annuel a toujours été négatif. Quelques remontées du solde ont été observées au début des années 1990 mais, depuis 1996-1997, la chute a repris de plus belle. La région perd présentement environ 3000 personnes par année au profit du reste de la province (surtout les régions de Québec et Montréal). Rappelons qu'il s'agit là d'un résultat net des mouvements migratoires interrégionaux et que le nombre total de sortants interrégionaux est beaucoup plus élevé que le solde. Selon l'Institut de la statistique du Québec (ISQ), 6636 résidents de la région du SLSJ sont allés s'établir ailleurs au Québec durant la seule année 2002-2003, pendant que 3779 résidents d'autres régions effectuaient le mouvement inverse, ce qui n'est tout de même pas négligeable. Quant aux autres types de migration, on peut constater que les gains et les pertes y sont à peu près équivalents, à part une brève exception du côté des migrations internationales au début des années 1990. Ainsi, l'évolution du solde migratoire total suit de très près celle du solde migratoire interrégional.

\section{Figure 5 - Soldes migratoires au Saguenay-Lac-Saint-Jean, 1986-2026 (projections à partir de 2003-2004)}

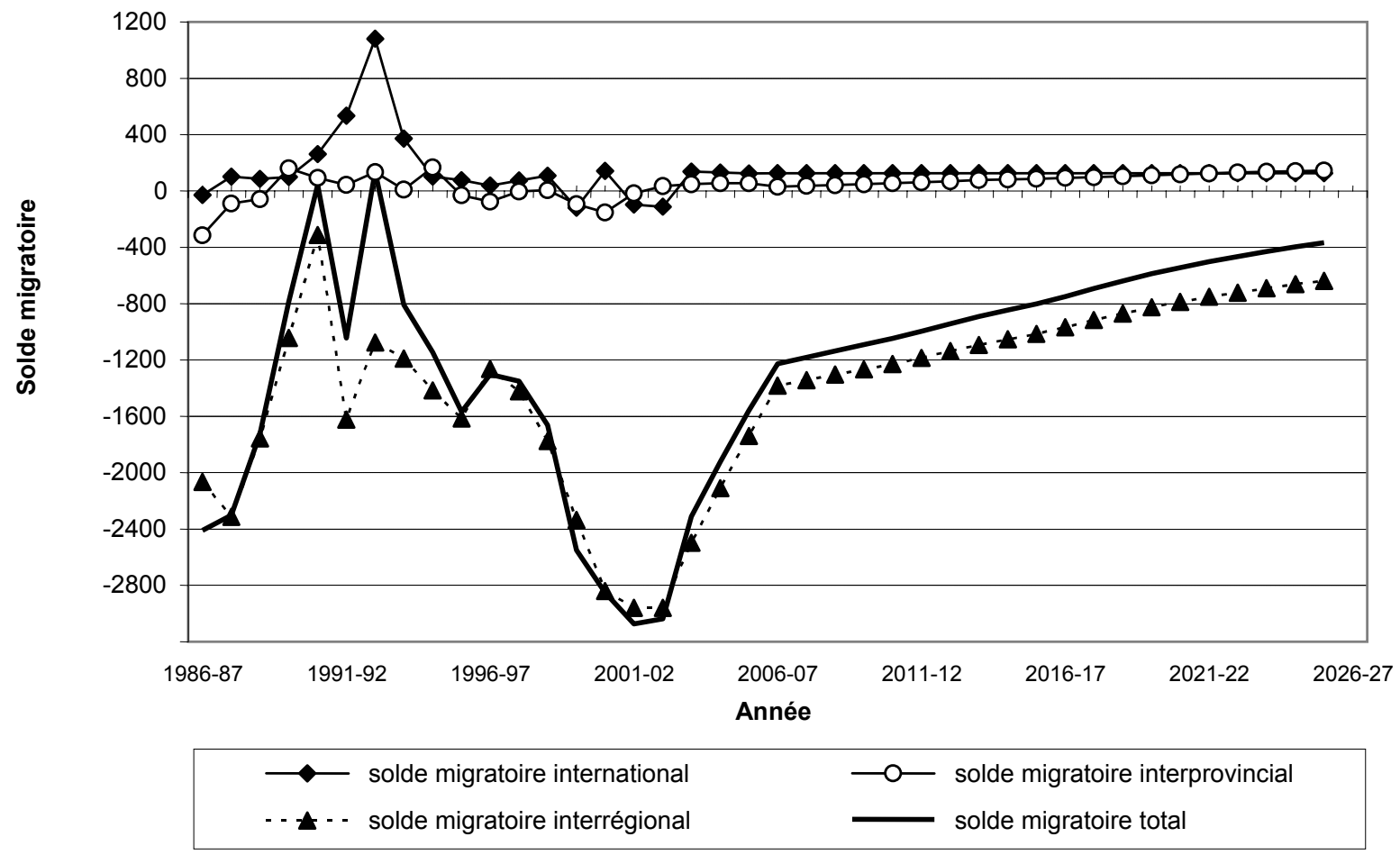

Mentionnons enfin que, bien que les migrations touchent toutes les tranches d'âge de la population, certains groupes sont particulièrement concernés. En
2002-2003, par exemple, près de $43 \%$ des entrées et sorties interrégionales ont été effectuées par des jeunes âgés entre 20 et 30 ans. Ce résultat n'est 
cependant pas propre à la population du SLSJ : de tout temps et dans la plupart des populations, la plus grande partie des migrations ont été le fait de jeunes adultes. Dans un contexte de faible accroissement naturel, cette perte de jeunes femmes et de jeunes hommes est particulièrement défavorable puisqu'elle entraîne aussi la perte des enfants qu'ont ou qu'auront ces jeunes.

\section{L'avenir démographique : si la tendance se maintient...}

L'ISQ produit et révise régulièrement des perspectives de population pour le Québec et ses régions. Ces perspectives s'appuient sur la méthode des composantes démographiques qui consiste à tenir compte de l'évolution de chacun des phénomènes responsables du renouvellement démographique, soit la fécondité, la mortalité et la migration. À partir d'hypothèses basées sur l'évolution récente de ces phénomènes, la population d'un territoire donné est soumise aux conditions démographiques prévues pour les années à venir. Habituellement, ces perspectives ne dépassent guère 40 ou 50 ans (au-delà de cette durée, les résultats deviennent trop hasardeux). Les perspectives démographiques pour la région du SLSJ, dont certains éléments apparaissent dans les figures $1,4,5$ et 6 , ont été établies sur la base des hypothèses les plus plausibles retenues par l'ISQ dans sa plus récente révision (édition 2003, mise à jour en avril 2004).

Le constat est clair: si la tendance se maintient, la population de la région continuera à décroître au cours des vingt prochaines années (et même au-delà). Les prévisions de l'ISQ donnent un effectif d'environ 250000 personnes en 2026, soit une baisse de $10 \%$ par rapport à la population actuelle (figure 1). Du même coup, le poids démographique de la région du SLSJ dans l'ensemble du Québec poursuivra lui aussi sa descente : d'une proportion de 3,8\% qu'elle constitue actuellement, la population saguenayenne ne représentera plus que 3,1\% de la population québécoise en 2026.

Cette baisse de la population résulte de deux éléments principaux: la chute continue de l'accroissement naturel, consécutive au maintien de faibles niveaux de fécondité et à la hausse du nombre de décès découlant $\mathrm{du}$ vieillissement de la population, et les pertes migratoires causées surtout par les échanges déficitaires avec les autres régions de la province. À remarquer que cette baisse pourrait être encore plus rapide si les résultats récents concernant les migrations interrégionales se prolongeaient sur une plus longue période que celle prévue par l'ISQ. En effet, les résultats de l'ISQ montrent que le déficit migratoire interrégional se résorbera de façon assez prononcée d'ici la fin de la décennie (figure 5). Ces résultats découlent en partie du fait que les perspectives sont basées sur les tendances de la période 1991-2001, mais aussi de la baisse prévue des effectifs chez les jeunes de 20-30 ans qui, comme nous l'avons vu, ont la probabilité la plus élevée de migrer.

\section{Si la tendance se maintient, la population de la région continuera à décroître au cours des vingt prochaines années.}

Cette remontée prévue du solde migratoire ne pourra cependant contrer les effets de la baisse de la fécondité. L'ISQ ne prévoit pas de remontée spectaculaire de la fécondité au cours des prochaines années et, avec un nombre moyen d'environ 1,5 enfant par femme, la population de pourra plus assurer son renouvellement par l'accroissement naturel. Puisque le nombre de décès ne pourra qu'augmenter (malgré la hausse continue de l'espérance de vie), on prévoit que, d'ici quelques années (soit vers 2011), il y aura dorénavant plus de décès que de naissances dans la population du SLSJ (figure 4). En 2025, on comptera environ 2900 décès et moins de 1800 naissances. Comme le faisait remarquer Chantal Girard (démographe à l'ISQ) lors du dernier congrès de l'Association des démographes du Québec ${ }^{6}$ : «À l'avenir, la population se rendra plus souvent au cimetière qu'à la pouponnière ».

Les conséquences et, en quelque sorte, l'aboutissement de ces perspectives démographiques se retrouvent dans la pyramide des âges de la population du SLSJ prévue pour l'année 2026 (figure 6). Il n'y a pas si longtemps, cette pyramide avait vraiment la forme d'une pyramide, avec une base élargie et des effectifs proportionnellement moins nombreux aux âges avancés. C'était le cas jusqu'au début des années 1960. Depuis ce temps, la base de la pyramide se rétrécit et les étages supérieurs prennent de l'ampleur. En 2026, la pyramide d'âge de la population du SLSJ aura plutôt l'allure d'une toupie. Les classes d'âge les plus nombreuses seront toujours celles des baby-boomers 
qui se retrouveront à ce moment-là parmi les 60-75 ans. Les générations nées après 1976 seront de moins en moins nombreuses. Les enfants de 0 à 14 ans (soit ceux qui naîtront entre 2011 et 2026) ne représente- ront que $12,5 \%$ de la population en 2026 . À titre comparatif, cette tranche d'âge composait $43 \%$ de la population de la région en 1961.

Figure 6 - Pyramide des âges de la population du Saguenay-Lac-Saint-Jean, $1^{\text {er }}$ juillet 2026

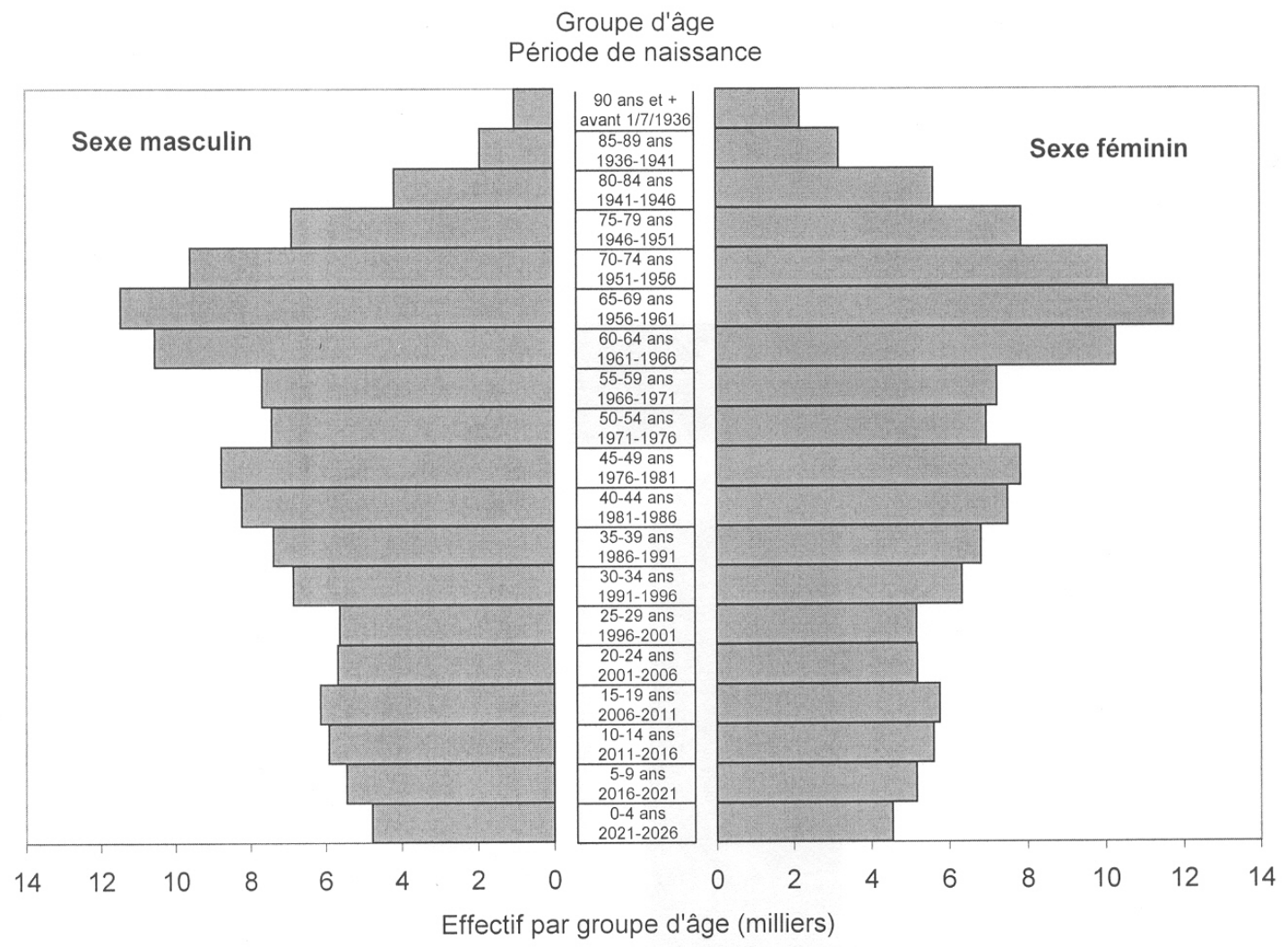

\section{Conclusion}

Depuis une dizaine d'années, la population du SLSJ décroît, comme quelques autres populations régionales du Québec, et tout indique que cette décroissance se poursuivra au cours des prochaines années. Cette situation de décroissance est souvent perçue de façon négative, et les plus pessimistes diront qu'à long terme, elle mènera inévitablement à la disparition de la population. Mais nous n'en sommes pas là. Même en prolongeant pendant 100 ans les tendances récentes, la région compterait encore 120000 habitants au début du $22^{\mathrm{e}}$ siècle, ce qui est encore plus élevé que les populations actuelles de la Gaspésie et de la CôteNord. Il n'y a donc pas lieu de s'alarmer outre mesure. On peut cependant s'interroger sur les moyens à prendre pour renverser la situation. Les deux phénomènes démographiques qui sont les plus susceptibles de bouger sont la fécondité et, surtout, la migration. Un redressement de la fécondité à un peu plus de 2 enfants par femme pourrait à tout le moins assurer une croissance non négative. Encore faudraitil que le solde migratoire devienne lui aussi à peu près nul. Mais, pour l'ensemble de la population québécoise, une remontée de l'indice synthétique de fécondité à des valeurs de 2,1 ou plus est peu probable, du moins à court terme. Les mesures mises de l'avant il y a une quinzaine d'années par le gouvernement provincial (les « bébés bonus »), dans le but de redresser la fécondité, ont été fort peu concluantes. Il faudrait la mise en place d'une politique familiale beaucoup plus dynamique, offrant un réel soutien aux couples et aux familles, pour espérer une remontée durable de la fécondité.

La solution la plus envisageable réside donc du côté de la migration. Pour freiner la décroissance et éventuellement retrouver une croissance positive, il faudra 
nécessairement que les échanges migratoires deviennent plus favorables à la région. Cela est évidemment plus facile à dire qu'à faire, surtout dans le contexte économique actuel. Mais on peut tout de même avancer quelques suggestions.

\section{Pour freiner la décroissance et éventuellement retrouver une croissance positive, il faudra nécessairement que les échanges migratoires deviennent plus favorables à la région.}

La première solution qui vient à l'esprit et dont on parle abondamment depuis quelques années serait de stopper le fameux « exode » des jeunes. Nous croyons cependant que la « rétention » ne représente au mieux qu'une petite partie de la solution. Nous avons vu en effet que les jeunes adultes sont ceux qui sont les plus susceptibles de migrer, peu importe les populations concernées. Il serait plutôt illusoire, et sans doute peu souhaitable, de vouloir à tout prix renverser ce phénomène. Les jeunes continueront à se déplacer, même dans un contexte économique plus favorable. D'ailleurs, il ne faut pas oublier que ce sont aussi, en bonne partie, des jeunes qui viennent (ou reviennent) s'installer dans la région, en provenance principalement des autres régions du Québec, mais aussi de l'étranger. On néglige trop souvent de prendre en compte ce mouvement inverse qui contribue à atténuer grandement les départs. Nous croyons que des efforts supplémentaires devraient être envisagés afin d'attirer encore plus ces entrants interrégionaux. Trop d'énergie est peut-être dépensée dans le but d'éviter les départs, comparativement à celle qui est fournie pour inciter les arrivées. D'un point de vue démogra- phique, ce n'est pas tant le nombre de sorties qu'il faut déplorer, mais le fait que le nombre d'entrées ne soit tout simplement pas aussi élevé.

Au fond, il s'agit de viser un certain équilibre dans les échanges migratoires avec les autres régions. Évidemment, les moyens qui permettraient d'atteindre cet équilibre ne relèvent pas de la démographie, mais plutôt de l'économie et surtout d'une volonté claire de la société québécoise de soutenir le développement régional.

\section{Notes et références}

1 Les auteurs possèdent tous deux un doctorat en démographie de l'Université de Montréal et sont professeurs au département des sciences humaines de l'Université du Québec à Chicoutimi. Leurs travaux de recherche portent principalement sur l'étude du rôle des comportements démographiques dans la structuration des bassins génétiques régionaux du Québec.

2 La plus grande partie des données sur l'évolution démographique récente provient du site Internet de l'Institut de la statistique du Québec (http://www.stat.gouv.qc.ca).

3 Les données sur l'histoire démographique du SLSJ ont été tirées de Pouyez C, Y. Lavoie, G. Bouchard, R. Roy, J.-P. Simard et M. St-Hilaire (1983). Les Saguenayens. Introduction à l'histoire des populations du Saguenay, $X V I^{e}-X X^{e}$ siècles, Québec, Presses de l'Université du Québec.

4 À ce sujet, on peut consulter l'ouvrage Histoire d'un génome, publié en 1991 sous la direction de Gérard Bouchard et Marc De Braekeleer aux Presses de l'Université du Québec (particulièrement les chapitres 7 et 8 ).

5 Pouyez C, Y. Lavoie, G. Bouchard, R. Roy, J.-P. Simard et M. St-Hilaire, op.cit.

6 Congrès de l'ACFAS, Montréal, mai 2004. 


\section{Publicité}

\section{Revue Organisations et territoires}

«Vous désirez recevoir un de nos numéros déjà parus ? " 